\title{
Wnt inhibitory factor-1 functions as a tumor suppressor through modulating Wnt/B-catenin signaling in neuroblastoma
}

Jiao Zhang, Bin Zhou, Yinghua Liu, Keling Chen, Pingqian Bao, Yi Wang, Jiaxiang Wang, Zongguang Zhou, Xiaofeng Sun and Yuan Li

\section{Linköping University Post Print}

\section{Tweet}

N.B.: When citing this work, cite the original article.

Original Publication:

Jiao Zhang, Bin Zhou, Yinghua Liu, Keling Chen, Pingqian Bao, Yi Wang, Jiaxiang Wang, Zongguang Zhou, Xiaofeng Sun and Yuan Li, Wnt inhibitory factor-1 functions as a tumor suppressor through modulating $\mathrm{Wnt} / \beta$-catenin signaling in neuroblastoma, 2014, Cancer Letters, (348), 1-2, 12-19.

http://dx.doi.org/10.1016/j.canlet.2014.02.011

Copyright: Elsevier

http://www.elsevier.com/

Postprint available at: Linköping University Electronic Press

http://urn.kb.se/resolve?urn=urn:nbn:se:liu:diva-106900 


\title{
Wnt Inhibitory Factor-1 functions as a tumor suppressor through modulating Wnt/ק-catenin signaling in neuroblastoma
}

\author{
Jiao Zhang ${ }^{1}$, Bin Zhou ${ }^{2}$, Yinghua Liu ${ }^{1}$, Keling Chen $^{2}$, Pingqian Bao ${ }^{1}$, Yi Wang ${ }^{1}$, Jiaxiang \\ Wang $^{3}$, Zongguang Zhou ${ }^{2,5}$, Xiaofeng Sun ${ }^{2,4}$, Yuan Li ${ }^{1,2 *}$ \\ ${ }^{1}$ Department of Pediatric Surgery, West China Hospital, Sichuan University, Chengdu, Sichuan, \\ 610041, China \\ ${ }^{2}$ Institute of Digestive Surgery, State Key Laboratory of Biotherapy, West China Hospital, \\ Sichuan University, Chengdu, Sichuan, 610041, China \\ ${ }^{3}$ Department of Pediatric Surgery, the First Affiliated Hospital of Zhengzhou University, \\ Zhengzhou, Henan, 450052, China \\ ${ }^{4}$ Division of Oncology, Department of Clinical and Experimental Medicine Faculty of Health \\ Sciences, Country Council of Östergötland, University of Linköping, Linköping S-581 85, \\ Sweden \\ ${ }^{5}$ Department of Gastrointestinal Surgery, West China Hospital, Sichuan University, Chengdu, \\ Sichuan, 610041, China \\ *Correspondence to: \\ Professor Yuan Li, M.D.,Ph.D \\ Department of Pediatric Surgery, West China Hospital, Sichuan University, Chengdu, Sichuan, \\ 610041, China \\ Tel: +86 18980601882; Fax: +86 028 85164036; E-mail: liyuanletters@163.com
}

Conflicting Interest: There are no potential conflicts of interest.

Key words: neuroblastoma, Wnt inhibitory factor-1 (WIF-1), Wnt/ $\beta$-catenin signaling, methylation

Abbreviations: WIF-1, Wnt inhibitory factor-1; MSP, methylation-specific PCR; 5-aza-dC, 5Aza-2'-deoxycytidine; IHC, Immunohistochemistry assay; IF, Immunocytofluorescence assay; ICC, Immunocytochemistry assay

Grand support: This work was supported by grants from the National Natural Science Fund of China (NSFC key project No.30830100; project No.30972924; project No.81170439). The Project was sponsored by the Scientific Research Foundation for the Returned Overseas Chinese Scholars, State Education Ministry of China (project No.20101174-4-2) and The Research Fund for the Doctoral Program of Higher Education, State Education Ministry of China (project No. 200806100058). 


\begin{abstract}
Neuroblastoma is the most common extracranial solid tumor in childhood and is associated with serious morbidity and mortality. The effective treatment of neuroblastoma remains one of the major challenges in pediatric oncology. The Wnt signaling pathway has been shown to play a significant role in the pathogenesis of adult and pediatric tumors. WIF-1 has been identified as an important Wnt antagonist which inhibits Wnt/ $\beta$-catenin signaling by directly binding to Wnt proteins. However, the expression and function of WIF-1 in neuroblastoma remains unknown. The present study showed that WIF-1 was downregulated with high level promoter methylation in neuroblastoma cells, and was significantly upregulated after exposure to demethylating agent. This finding suggests that downregulation of WIF-1 was associated with its promoter methylation in neuroblastoma. To further study the potential function of WIF-1 in neuroblastoma, we constructed a plasmid that over-expressed WIF-1 and transfected the plasmid into one neuroblastoma cell line SK-N-SH. We found that restoration of WIF-1 inhibited the growth and proliferation of neuroblastoma cells in vitro. Morever, Wnt/ $\beta$-catenin signaling activity and target genes expression were reduced by WIF-1 restoration. These results provide support that WIF-1 is downregulated and functions as a tumor suppressor by antagonizing Wnt/ $\beta$-catenin signaling in neuroblastoma, suggesting a potential role as a therapeutic target in neuroblastoma.
\end{abstract}




\section{Introduction}

Neuroblastoma is the most common form of pediatric cancer, accounting for approximately $30 \%$ of all infantile malignancies and $15 \%$ of all pediatric oncology deaths [1]. Patients with neuroblastoma often present with advanced disease characterized by invasive tumor masses and/or metastases to distant organs [2]. With the development of molecular biology and recognition to neuroblastoma, researchers have begun to seek specific regulators for carcinogenic signaling pathways as new therapeutic strategies.

The Wnt/ $\beta$-catenin signaling pathway is a highly conserved signaling pathway during evolution. It plays an essential role in regulating embryonic development, cell proliferation, and differentiation [3]. A large body of evidence indicates that abnormal $\mathrm{Wnt} / \beta$-catenin pathway activity is associated with many types of human tumor [4-6]. Other studies have also shown that aberrant activation of this pathway is induced by dysregulation of Wnt pathway members or by downregulation of endogenous Wnt antagonists [7-9]. Aberrant expression of $\mathrm{Wnt} / \beta$ catenin signaling is known to play a significant role in various processes of early development and in the pathogenesis of adult and pediatric tumors including neuroblastoma $[10,11]$.

Constitutive activation of the Wnt/ $\beta$-catenin signaling pathway can be induced by dysregulation of Wnt pathway members, such as overexpression of $\beta$-catenin $[4,6]$. Other workers have shown that dysregulated Wnt signaling can be experimentally induced by Wnt antagonists such as Dickkppfs and sFRPs $[8,9]$.

Wnt inhibitory factor-1 (WIF-1) is an important antagonist involved in the Wnt/ $\beta$-catenin signaling pathway. It is highly conserved between species and was initially identified from the human retina [12]. WIF-1 secretion has been shown to inhibit the upstream of Wnt/ $\beta$-catenin signal transduction by directly binding to Wnt proteins in the extracellular space [13]. This prevents the activation of Wnt target genes including c-myc and cyclin D1 [14-15]. Downregulation of WIF-1 has been reported in many human malignancies [16-21]. However, the expression and functional significance of WIF-1 in neuroblastoma remains unknown.

\section{Materials and Methods}

\subsection{Tumor Samples and cell lines}

Tumor samples including 42 neuroblastoma tissues, 14 ganglioneuroma tissues and 11 normal adrenal gland tissues were obtained from Department of Pathology of the West China Hospital of the Sichuan University, China.

Three human neuroblastoma cell lines IMR-32, SK-N-SH, SH-SY5Y were used as experimental cell lines, and a human embryonic kidney cell line HEK293 was used as normal control. SK-N-SH and HEK293 were purchased from the American Type Culture Collection (ATCC, Manassas, VA). IMR-32 and SH-SY5Y were purchased from Cell Bank of the Chinese Academy of Sciences (Shanghai, China).

IMR-32 cells were cultured in EMEM (Invitrogen, Carlsbuel, CA) supplemented with 10\% fetal bovine serum (FBS). SK-N-SH and HEK293 cells were cultured in DMEM (Invitrogen) supplemented with 10\% FBS. SH-SY5Y was cultured in RPMI-1640 (Invitrogen) supplemented with $10 \%$ FBS. All cells were cultured at $37{ }^{\circ} \mathrm{C}$ in a humidified incubator with $5 \% \mathrm{CO}_{2}$. 


\subsection{Demethylation}

Cultured cell lines were seeded into six-well plates at a density of $1 \times 10^{5}$ cells per well. Twenty-four hours later, cells were exposed to $50 \mu \mathrm{mol} / \mathrm{L}$ of the demethylating agent 5 -aza-dC (Sigma, St. Louis, MO) for 4 days. Fresh demethylating agent was added every $12 \mathrm{~h}$. Cells not treated with 5-aza-dC were used as controls. Genomic DNA and RNA were extracted from the cells at the end of the experiment.

\subsection{Immunohistochemistry}

Immunohistochemical staining (IHC) was used to determine WIF-1 expression in tissues. WIF-1 antibody (1:100; Epitomics, Burlingame, CA) and diaminobenzidine (DAB) were used for staining and visualization, respectively. Normal adrenal gland tissues were used as positive controls.

Results were independently evaluated by two pathologists in a blind fashion. IHC staining was graded on an arbitrary scale from 0 to 2 : where 0 represented negative expression $(0-20 \%$ positive cells), 1 represented weakly positive expression (20-50\% positive cells), and 2 represented strongly positive expression (50-100\% positive cells). The scale was determined according to the average number of positive cells in 10 random fields of one slide [22].

\subsection{RNA extraction and real-time PCR}

Total RNA was isolated using the Trizol reagent (Invitrogen) according to the manufacturer's protocol. cDNA was synthesized using a First Strand cDNA Synthesis kit (Fermentas, Burlington, Ontario) according to the manufacturer's protocol. Real-time quantitative RT-PCR (qRT-PCR) was performed using a relative quantification protocol on an iCycler iQ System (BioRad Laboratories, Hercules, CA). $\beta$-actin was used as an internal control. RNA extracted from HEK293 cells served as a positive control, and reagent without template served as a negative control.

The relative fold change in mRNA expression compared with control was calculated using the comparative $\mathrm{C}_{t}$ method [23]. The sequences of PCR primers are listed in Supplimental Table 1.

\subsection{Protein extraction and Western blotting}

Total cell proteins were isolated and purified using the Total Protein Kit (Sigma) according to the manufacturer's protocol. The protein content was determined using the BCA assay method (Pierce, Rockford, IL) with GAPDH was as an internal control. Briefly, SDS-PAGE 10\% separating gels were run using $20 \mu \mathrm{g}$ of each protein sample. After gel electrophoresis, protein extracts were transferred to polyvinylidene difluoride (PVDF) membrane (Bio-Rad, Hercules, $\mathrm{CA}$ ) and then blocked with 5\% BSA at room temperature for $1 \mathrm{~h}$. The membranes were then incubated overnight at $4^{\circ} \mathrm{C}$ with antibody against WIF-1 (1:100, Epitomics), $\beta$-catenin (1:5000, Epitomics), c-myc (1:2000, Epitomics), cyclinD1 (1:10000, Epitomics) or GAPDH (1:1000, Cell Signaling, Beverly, MA). After washing, the membranes were incubated with secondary antibody (1:2000, Cell Signaling, Beverly, MA) at room temperature for $2 \mathrm{~h}$.

The membranes were visualized using the Bio-Rad ChemiDoc XRS imaging system. Densitometric analyses were performed using BioRad Quantity One software. The intensity of the bands of each treatment was compared with the intensity of control.

\subsection{Genomic DNA extraction and methylation-specific PCR (MSP)}


Genomic DNA was extracted from neuroblastoma cell lines and HEK293 cells using Blood and Cell Culture DNA mini kit (Qiagen, Valencia, CA) according to the manufacturer's protocol. Bisulfite modification of genomic DNA was performed using the EZ DNA methylation kit (Zymo Research, Orange, CA) following the manufacturer's protocol. Bisulfitetreated genomic DNA was amplified using either a methylation-specific or an non-methylationspecific primer set. Fragments of MSP and USP were $145 \mathrm{bp}$ and $151 \mathrm{bp}$, respectively. The sequences of MSP primers are listed in Supplimental Table 1.

\subsection{Plasmid construction and cells transfection}

The full length cDNA of WIF-1 (Invitrogen) was subcloned into the HindIII-XhoI sites of pcDNA3.1 vector (Invitrogen) to generate pcDNA3.1-WIF-1 with confirmed sequence and orientation. SK-N-SH cells $\left(1 \times 10^{5}\right.$ cells $)$ were dissociated into single cell suspension and plated in 6-well plates for transfection. One day later, cells were transiently transfected with $5 \mu \mathrm{g}$ pcDNA3.1-WIF-1 or pcDNA3.1 (vector control) using Lipo 2000 (Invitrogen) according to the manufacturer's protocol. Forty-eight hours later, the stable transfected cells were selected for culture for 30 days with G418 $(700 \mu \mathrm{g} / \mathrm{ml})$. The expression of WIF-1 was assessed by Western blotting and real-time PCR. The selected stable transfected cells were cultured in DMEM containing 10\% FBS and G418 (500 $\mu \mathrm{g} / \mathrm{ml})$.

\subsection{Proliferation assay}

SK-N-SH cells $\left(5 \times 10^{3}\right.$ per well) were plated in 96 -well plates $24 \mathrm{~h}$ before transfection. After transfection with pcDNA3.1-WIF-1 or pcDNA3.1, cells were incubated for $144 \mathrm{~h}$. Cell viability was determined every $24 \mathrm{~h}$ by using the CellTiter 96 AQueous One Solution Cell Proliferation Assay (MTS assay) (Promega, Madison, WI) according to the manufacturer's protocol. Absorbance at $490 \mathrm{~nm}$ was measured with a microplate reader (BioRad) and used as a measure of cell number. The samples were repeated three times in triplicate.

\subsection{Soft Agar Colony Formation Assay}

Soft agar colony formation assay performed using 6-well plates. Each well contained $2 \mathrm{~mL}$ of $0.8 \%$ agar in complete medium as the bottom layer, $1 \mathrm{~mL}$ of $0.35 \%$ agar in complete medium with $6 \times 10^{3}$ stable transfected cells or vector control as the feeder layer, and $1 \mathrm{~mL}$ complete medium as the top layer. Cultures were maintained for 2 weeks under standard conditions. The number of colonies was determined with an inverted phase-contrast microscope at 100x magnification. Colonies containing more than 10 cells were counted.

\subsection{Dual-luciferase reporter assay}

TOP flash or FOP flash plasmids were cotransfected with either pcDNA3.1-WIF-1 or empty vector, together with an internal control pRL-TK vector using Lipo 2000. Forty-eight hours later, cells were harvested and analyzed for Firefly and Renilla luciferase activity using the Dual-Luciferase reporter assay kit (Promega). Each experiment was repeated three times.

\subsection{Immunocytochemistry assay}

SK-N-SH cells were dissociated into a single cell suspension and plated on coverslips. One day later, cells were transfected with pcDNA3.1-WIF-1 or empty vector. Forty-eight hours later, the transfected cells were fixed with $4 \%$ paraformaldehyde solution at room temperature for 30 minutes. Primary antibody and DAB were for staining and visualization, respectively. Cells were counterstained with hematoxylin. The primary antibodies were WIF-1 (1:100; 
Epitomics, Catalog\#T0809), $\beta$-catenin (1:250; Epitomics, Catalog\#1247-1), c-myc (1:250; Epitomics, Catalog\#1472-1) or cyclin D1 (1:100; Epitomics, Catalog\#2261-1).

\subsection{Immunocytofluorescence assay}

Transfected cells were fixed with $4 \%$ paraformaldehyde for 30 minutes and permeabilized with $0.2 \%$ Triton X-100 for 5 minutes. They were then blocked with 5\% normal goat serum for $1 \mathrm{~h}$ and incubated at $4{ }^{\circ} \mathrm{C}$ overnight with antibody against WIF-1, $\beta$-catenin, c-myc or cyclin $\mathrm{D} 1$ at the same concentrations used in ICC. After washing, the cells were incubated with FITCconjugated secondary antibodies (1:300; Sigma) at room temperature for $1 \mathrm{~h}$. Nuclei were stained with DAPI diluent (300nM; Sigma) at room temperature for 5 minutes. Localization of WIF-1, $\beta$-catenin, c-myc, and cyclin D1 was determined under Laser Scanning Confocal Microscope (Olympus, Tokyo, Japan) using $488 \mathrm{~nm}$ (FITC) and $358 \mathrm{~nm}$ (DAPI) excitation wavelengths.

\subsection{Statistical analysis}

Statistical analyses were performed using SPSS version 16.0 software for Windows. Data are shown as means and standard deviations (mean $\pm \mathrm{SD}$ ). Between group differences were analyzed using Student's t test. One-way ANOVA was used for multiple comparisons. Values of $P<0.05$ were considered statistically significant.

\section{Results}

\subsection{WIF-1 Protein expression is downregulated in neuroblastoma tissue samples}

Immunohistochemistry assay was performed to detect WIF-1 expression in 42 neuroblastoma tissues, 14 ganglioneuroma tissues, and 11 normal adrenal gland tissues (Fig. 1A). The mean expression scores for WIF-1 were $1.963 \pm 0.056,1.176 \pm 0.106$ and $0.470 \pm 0.275$ in normal adrenal gland tissues, ganglioneuroma and neuroblastoma cells, respectively. WIF-1 protein levels were downregulated in ganglioneuroma and neuroblastoma cells compared to the normal adrenal gland tissues (all $P<0.001$, Fig. 1B). Multiple comparison analysis indicated that the protein expression differences for WIF-1 within three tissues were statistically significant $(F=219.451, P<0.001)$.

\subsection{WIF-1 mRNA expression is downregulated in neuroblastoma cell lines}

qRT-PCR assay was used to analyze the mRNA expression of WIF-1 in three human neuroblastoma cell lines including IMR-32, SK-N-SH and SH-SY5Y cells. HEK293 cells served as the positive control. In the three neuroblastoma cells, IMR-32 has the worst differentiation and highest malignance, SH-SY5Y has best differentiation and lowest malignance, SK-N-SH has middle differentiation and malignance [24, 25]. The mRNA expression level of HEK293 was set as 100\% (as control), comparison was made between control and three neuroblastoma cells respectively. The relative mean mRNA expression of WIF-1 was $29.341 \pm 3.594 \%, 37.278 \pm 5.223 \%$ and $69.196 \pm 9.381 \%$ respectively in IMR-32, SK$\mathrm{N}-\mathrm{SH}$ and SH-SY5Y cells. The results showed that WIF-1 mRNA levels were downregulated in all three neuroblastoma cell lines compared to HEK293 (all $P<0.001$, Fig. 1C). Multiple comparison analysis indicated that the mRNA expression differences in WIF-1 expression between the three neuroblastoma cell lines were statistically significant $(F=58.691, P<0.001)$. 


\subsection{WIF-1 Protein expression is downregulated in neuroblastoma cell lines}

Western blotting was performed to analyze protein expression of WIF-1 in three human neuroblastoma cell lines and HEK293 cells. The protein expression level of HEK293 was set as $100 \%$ (as control), comparison was made between control and three neuroblastoma cells respectively. The relative mean protein expression of WIF-1 was $17.891 \pm 2.316 \%$, $20.475 \pm 4.563 \%$ and $45.269 \pm 4.934 \%$ in IMR-32, SK-N-SH and SH-SY5Y cells, respectively. Results showed that WIF-1 protein levels were downregulated in the three neuroblastoma cell lines compared to HEK293 (all $P<0.001$, Fig. 1D and 1E). Multiple comparison analysis showed statistically significant differences in WIF-1 expression between the three neuroblastoma cell lines $(F=73.472, P<0.001)$.

\subsection{High methylation status of the WIF-1 promoter region in neuroblastoma cell lines}

To study the potential mechanism of WIF-1 mRNA downregulation in neuroblastoma, we examined the methylation status of WIF-1 promoter in four cell lines. WIF-1 promoter region has been identified and described previously [26]. MSP results showed that the relative levels of WIF-1 promoter methylation in neuroblastoma cell lines were all significantly higher than in HEK293 cells (Fig. 2A). In addition, the relative level of WIF-1 promoter methylation was inversely related to the mRNA level of WIF-1 in neuroblastoma cells.

\subsection{Restoration of WIF-1 expression after 5-Aza-dC treatment}

Protein and mRNA levels of WIF-1 were detected to identify whether the silencing of WIF-1 was associated with its promoter methylation. Neuroblastoma cells and HEK293 cells were exposed to 5-Aza-dC to observe the restoration of WIF-1 expression. WIF-1 baseline expression for each cell line (before 5-Aza-dC treatment) was set as $100 \%$ (as control), comparison was made before and after 5-Aza-dC treatment respectively. The results of qRTPCR showed that mRNA expression of WIF-1 was upregulated in neuroblastoma cells exposed to 5-Aza-dC (Fig. 2B). Western blotting analysis showed increased protein expression of WIF1 in neuroblastoma cells (Fig. 2C. 2D). The extent of upregulation was related to the relative level of WIF-1 promoter methylation, and inversely related to the mRNA level of WIF-1 in neuroblastoma cells.

\subsection{Restoration of WIF-1 gene by constructed WIF-1 expression plasmid}

A WIF-1 expression plasmid pcDNA3.1-WIF-1 was successfully constructed and transfected into SK-N-SH cells. Real-time PCR and western blotting confirmed WIF-1 was significantly overexpressed in SK-N-SH cells transfected with pcDNA3.1-WIF-1(SK-N$\mathrm{SH} / \mathrm{WIF}-1$ ) compared to cells transfected with the empty vector (SK-N-SH/vector) (all $P<0.01$; Figure 3A). ICC analysis showed stronger cytoplasmic and membranous staining with SK-N$\mathrm{SH} / \mathrm{WIF}-1$ than in SK-N-SH/vector (Supplimental Fig. 1A). Immunofluorescence analysis also showed enhanced inflorescent staining in SK-N-SH/WIF-1 compared to negative control (Fig. $4 \mathrm{~A})$.

\subsection{Inhibitory effects of WIF-1 on neuroblastoma cell growth and proliferation}

The effects of WIF-1 restoration on neuroblastoma cell growth and proliferation were investigated using cell proliferation and colony formation assays. In the cell proliferation assay, SK-N-SH/WIF-1 showed significant inhibition of growth compared with SK-N-SH/vector $(P$ $<0.01$ for Days 4-6; Fig. 5A). In the colony forming assay, SK-N-SH/WIF-1 showed significant 
reduction of colony numbers compared with SK-N-SH/vector ( $P=0.002$; Fig. 5C, 5D).

\section{8. $\beta$-catenin Protein expression is upegulated in neuroblastoma cell lines}

A
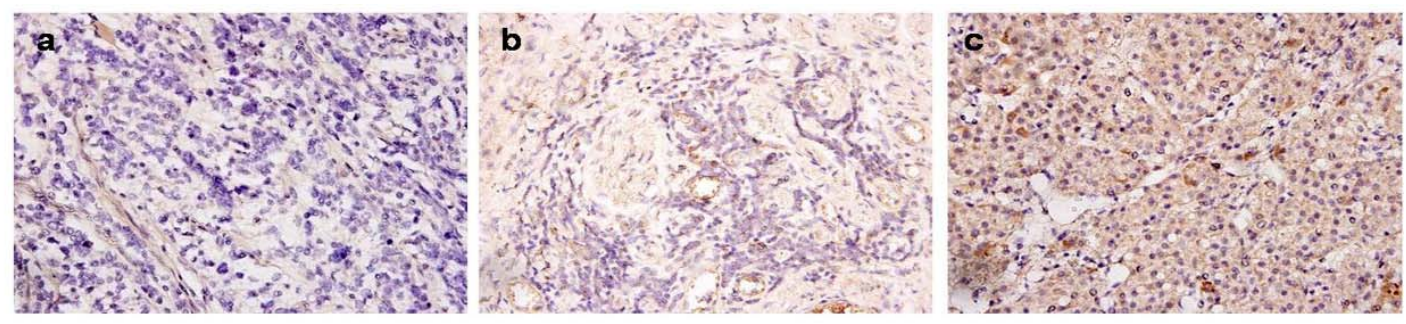

B

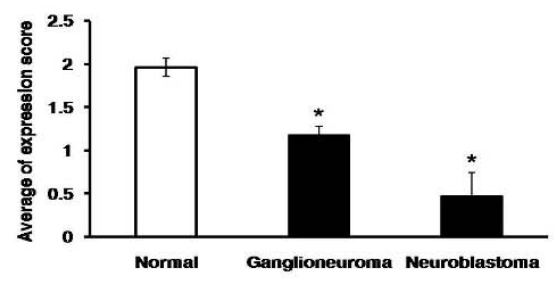

C

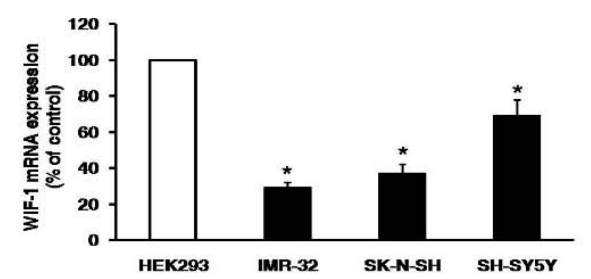

$\mathbf{F}$

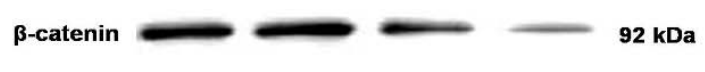

GAPDH

G

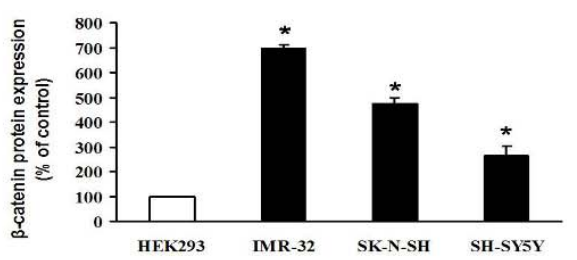

D

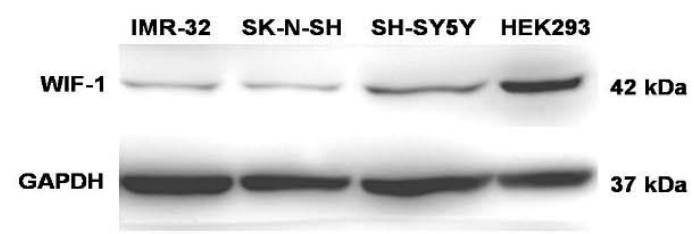

E

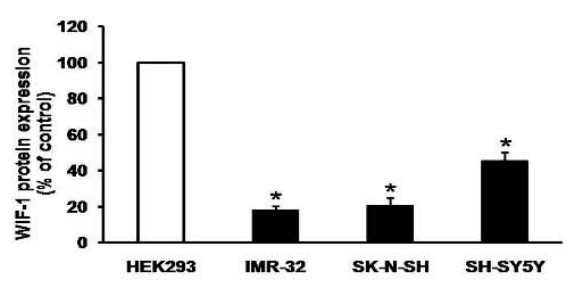

H

IMR-32 SK-N-SH SH-SY5Y HEK293

Cyclin D1

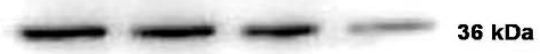

GAPDH

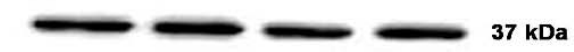

I

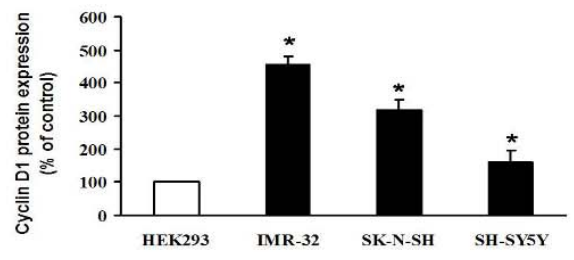

Fig. 1. WIF-1 expression in 67 tissues samples and four cell lines. The 67 tissue samples included 42 neuroblastomas tissues, 14 ganglioneuroma tissues and 11 normal adrenal gland tissues. The Four cell lines included three human neuroblastoma cell lines IMR-32, SK-N-SH, SH-SY5Y and one control cell line HEK293. (A) Protein levels of WIF-1 were detected by immunohistochemistry assay ( $\times 400$ magnification $)$ in a: neuroblastoma, b: ganglioneuroma; c: normal adrenal gland samples. (B) There were differences in average expression IHC score in the three tissue types $(* P<0.001$, compared with the normal adrenal gland tissues). (C) Relative mRNA expression of WIF-1 normalized to $\beta$-actin in neuroblastoma and control cell lines HEK293 determined by qRT-PCR $(* P<0.01$, compared with HEK293 respectively). (D) Expression of WIF-1 proteins detected by Western blotting. (E) The relative WIF-1 protein level normalized to GAPDH assessed by BioRad Quantity One software $\left({ }^{*} P<0.001\right.$, compared with HEK293). (F) Expression of $\beta$-catenin proteins detected by Western blotting. (G) The relative $\beta$-catenin protein level normalized to GAPDH assessed by BioRad Quantity One software ( ${ }^{*} P<0.001$, compared with HEK293). (H) Expression of cyclin D1 proteins detected by Western blotting. (I) The relative cyclin D1 protein level normalized to GAPDH assessed by BioRad Quantity One software $\left({ }^{*} P<0.001\right.$, compared with HEK293). 
Western blotting was performed to analyze protein expression of $\beta$-catenin in three human neuroblastoma cell lines and HEK293 cells. The protein expression of $\beta$-catenin in HEK293 was set as control $(100 \%)$, and the protein expressions of three neuroblastoma cells were compared respectively with HEK293. The relative mean protein expression of $\beta$-catenin was $697.425 \pm 12.316 \%, 473.238 \pm 24.563 \%$ and $264.526 \pm 37.421 \%$ in IMR-32, SK-N-SH and SHSY5Y cells, respectively. Results showed that $\beta$-catenin protein levels were upregulated in the three neuroblastoma cell lines compared to HEK293 (all $P<0.001$, Fig. $1 \mathrm{~F}$ and 1G). Multiple comparison analysis showed statistically significant differences in $\beta$-catenin expression between the three neuroblastoma cell lines $(F=68.252, P<0.001)$.

\subsection{Cyclin D1 Protein expression is upegulated in neuroblastoma cell lines}

Western blotting was performed to analyze protein expression of cyclin D1 in three human neuroblastoma cell lines and HEK293 cells. The protein expression of cyclin D1 in HEK293 was set as control $(100 \%)$, and the protein expressions of three neuroblastoma cells were compared respectively with HEK293. The relative mean protein expression of cyclin D1 was $457.258 \pm 22.349 \%, 317.453 \pm 31.173 \%$ and $163.251 \pm 32.211 \%$ in IMR-32, SK-N-SH and SHSY5Y cells, respectively. Results showed that cyclin D1 protein levels were upregulated in the three neuroblastoma cell lines compared to HEK293 (all $P<0.001$, Fig. 1H and 1I). Multiple comparison analysis showed statistically significant differences in cyclin D1 expression between the three neuroblastoma cell lines $(F=72.537, P<0.001)$.

A
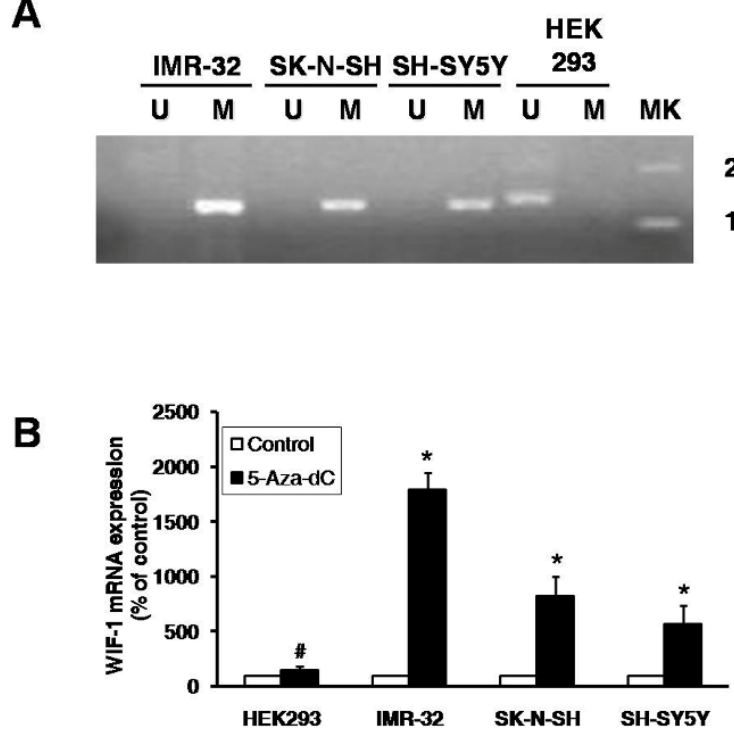

C

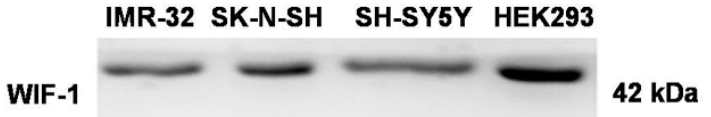

200 bp 100 bp

37 kDa

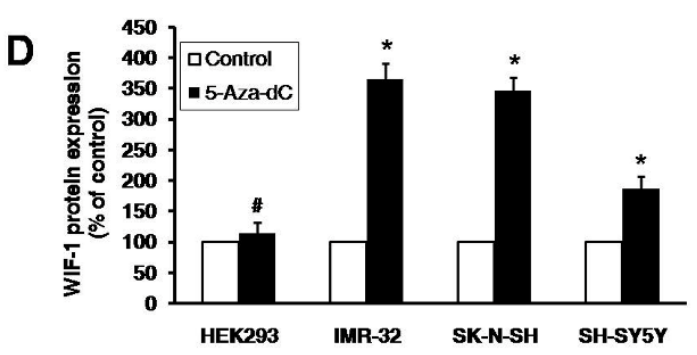

Fig. 2. Methylation analysis of WIF-1 promoter in four cell lines. (A) MSP analysis of WIF-1 promoter methylation in neuroblastoma cell lines and HEK293. Bands (151bp) in lanes labeled 'U' are unmethylated DNA product amplified with unmethylation-specific primers. Bands (145bp) in lanes labeled ' $\mathrm{M}$ ' are methylated DNA product amplified with methylation-specific primers. (B) Expression of WIF-1 mRNA levels in four cell lines after exposure to 5-Aza-dC as determined by qRT-PCR ( ${ }^{P}<0.01$, WIF-1 baseline expression for each cell line (before 5 -Aza-dC treatment) was set as $100 \%$ (as control), comparison was made before and after 5-Aza-dC treatment respectively). (C) Expression of WIF1 proteins in cell lines after treatment with 5 -Aza-dC detected by Western blotting. (D) The relative WIF-1 protein expressions after treatment with 5-Aza-dC assessed by BioRad Quantity One software $(* P<0.01$, WIF-1 baseline expression for each cell line (before 5-Aza-dC treatment) was set as 100\% (as control), comparison was made before and after 5-Aza-dC treatment respectively). 
A a

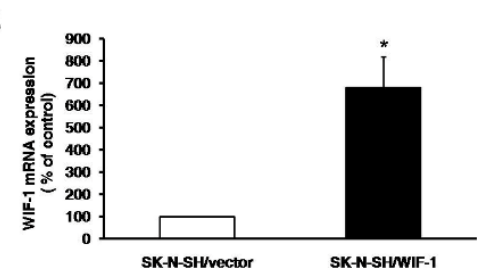

B a

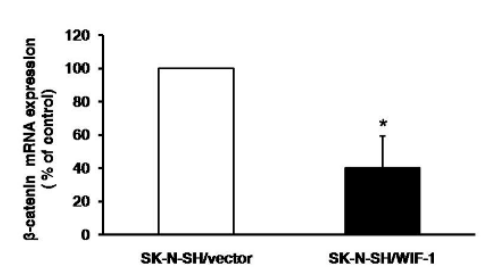

C a

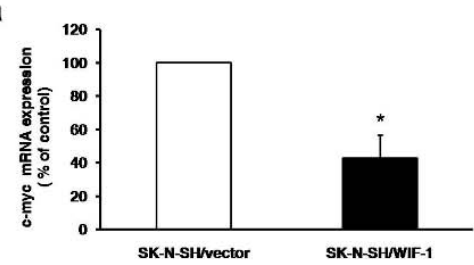

D a

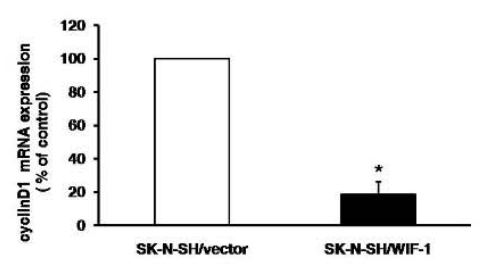

b

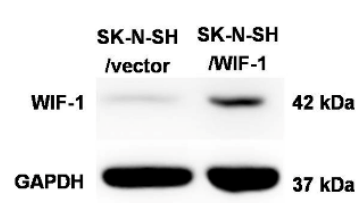

b

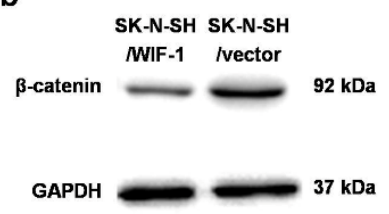

b

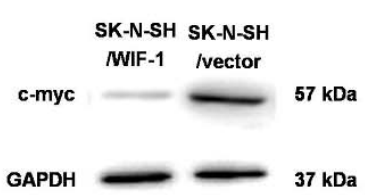

b

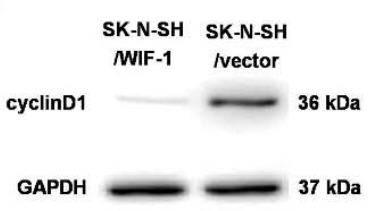

c
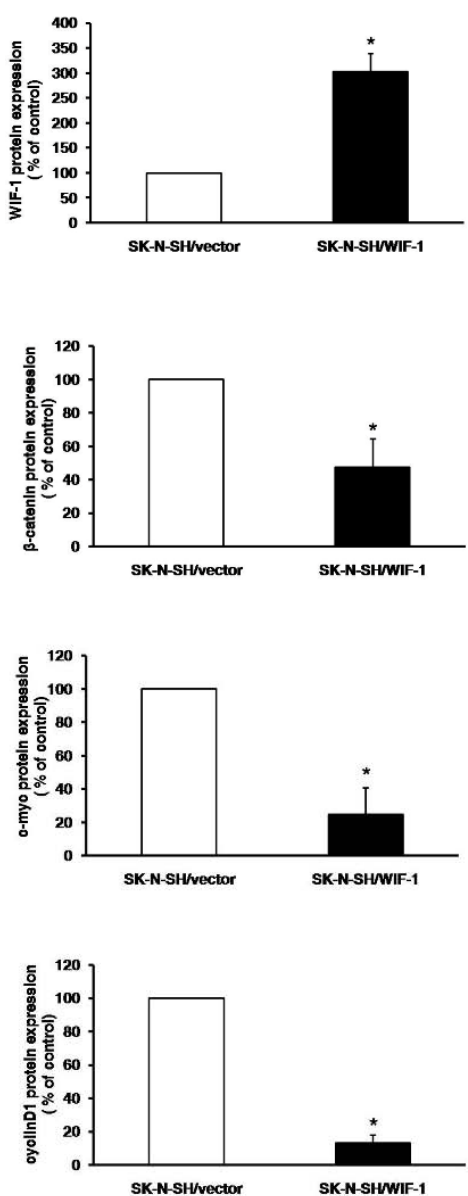

Fig. 3. mRNA and protein expression levels of WIF-1, $\beta$-catenin, c-myc and cyclin D1 in WIF-1/vector transfected SK$\mathrm{N}-\mathrm{SH}$ cells (* $\mathrm{P}<0.01$; a: mRNA expression; b: protein expression; c: analysis of gray-level values in western blotting). (A) WIF-1 expression was upregulated in WIF-1-transfected cells compared with vector control. (B) $\beta$-catenin expression was downregulated in WIF-1-transfected cells compared with vector control. (C) c-myc expression was downregulated in WIF-1-transfected cells compared with vector control. (D) Cyclin D1 expression was downregulated in WIF-1-transfected cells compared with vector control.

\subsection{Downregulation of Wnt/ $\beta$-catenin signaling activity by WIF-1 restoration}

A dual-Luciferase reporter assay was performed to analyze whether WIF-1 could inhibit the Wnt/ $\beta$-catenin pathway and reduce the transcriptional activity of the TCF- $\beta$-catenin complex in neuroblastoma cells [27]. The colorectal adenocarcinoma cell line Caco2, which has an activated $\mathrm{Wnt} / \beta$-catenin pathway, was used as positive control [28]. Results showed that ratios of TOP/FOP activity in Caco2, SK-N-SH/vector and SK-N-SH/WIF-1 were 12.35 \pm 3.60 , $8.57 \pm 1.53$ and $2.23 \pm 1.32$, respectively. Differences in the ratios of TOP/FOP activity between SK-N-SH/vector and SK-N-SH/WIF-1 were statistically significant ( $P=0.006$, Fig. 5B). These results suggest that WIF-1 restoration may inhibit the activity of $\mathrm{Wnt} / \beta$-catenin pathway in neuroblastoma cells.

\subsection{Downregulation of $\beta$-catenin expression by WIF-1 restoration}

We next investigated $\beta$-catenin expression in transfected cells as $\beta$-catenin is a key molecule in the Wnt signaling pathway. The result of qRT-PCR and Western blotting showed that $\beta$-catenin was significantly downregulated in WIF-1-transfected cells compared with 
vector controls (all $P<0.01$, Fig. 3B). ICC analysis showed weaker cytoplasmic and nuclear staining of $\beta$-catenin in SK-N-SH/WIF-1 than control cells (Supplimental Fig. 1B). IF analysis showed decreased cytoplasmic and nuclear inflorescent staining of $\beta$-catenin in SK-N-SH/WIF1 than in negative controls (Fig. 4B).

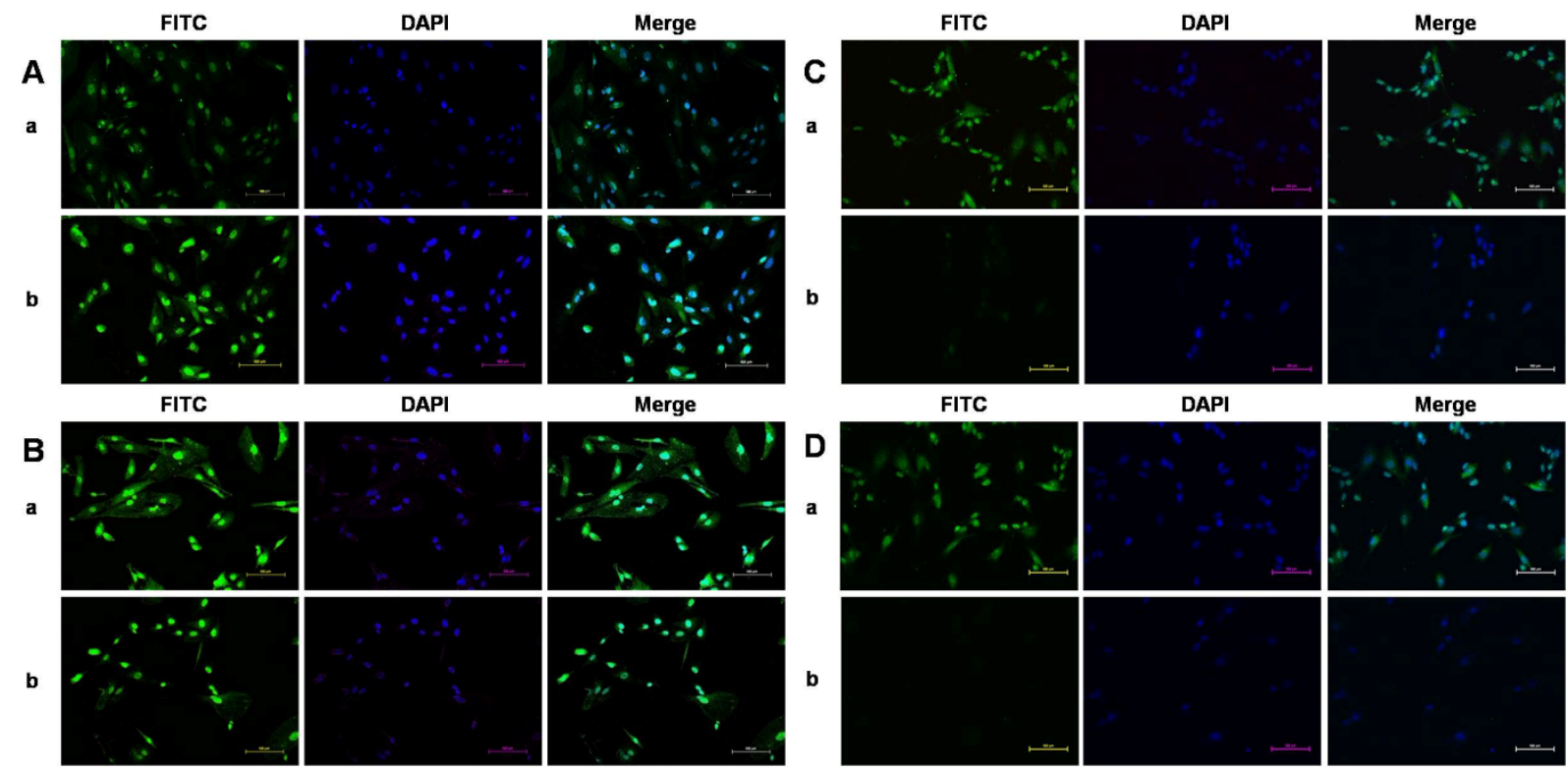

Fig. 4. Immunocytofluorescence staining of WIF-1, $\beta$-catenin, c-myc and cyclin D1 in WIF-1/vector transfected SK-NSH cells analyzed under laser scanning confocal microscope at $488 \mathrm{~nm}$ (FITC, green) and 358nm (DAPI, blue) excitation wavelengths (Scale bar $=100 \mu \mathrm{m}$; a: SK-N-SH/vector; b: SK-N-SH/WIF-1). (A) WIF-1 expression was upregulated with strong fluorescent dyes in SK-N-SH/WIF-1 cells. (B) The expression of $\beta$-catenin was downregulated with weak cytoplasmic and nuclear fluorescent dyes in SK-N-SH/WIF-1 cells. (C) The expression of c-myc was downregulated with weak cytoplasmic and nuclear fluorescent dyes in SK-N-SH/WIF-1 cells. (D) The expression of cyclin D1 was downregulated with weak cytoplasmic and nuclear fluorescent dyes in SK-N-SH/WIF-1 cells. 
A

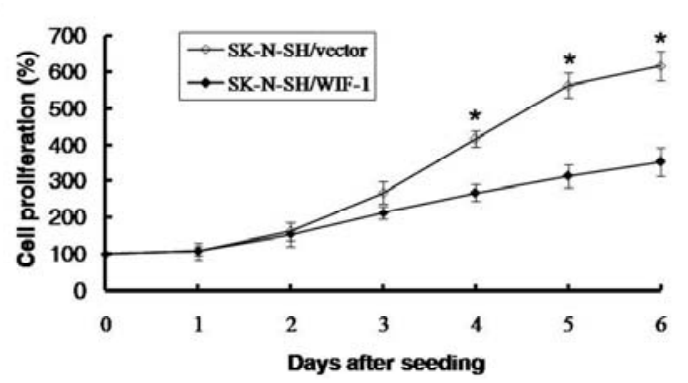

B

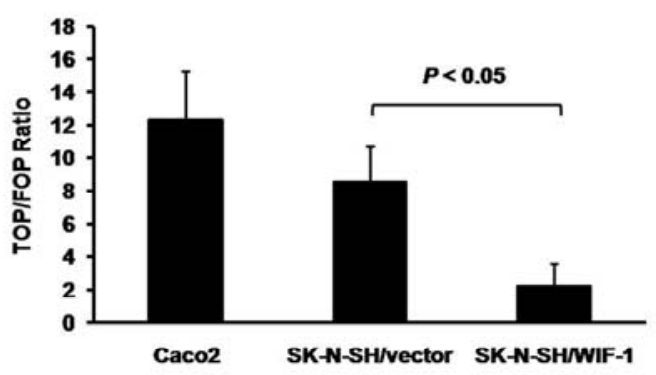

C

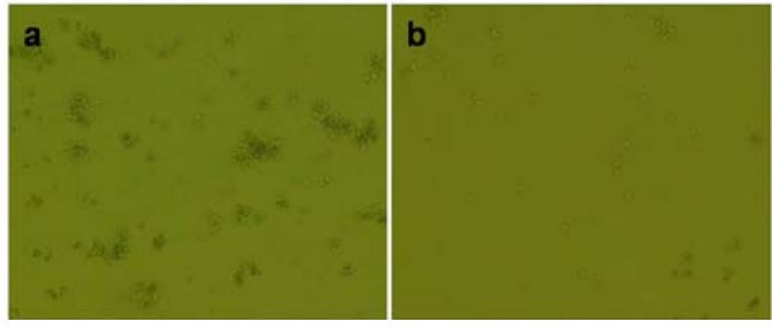

D

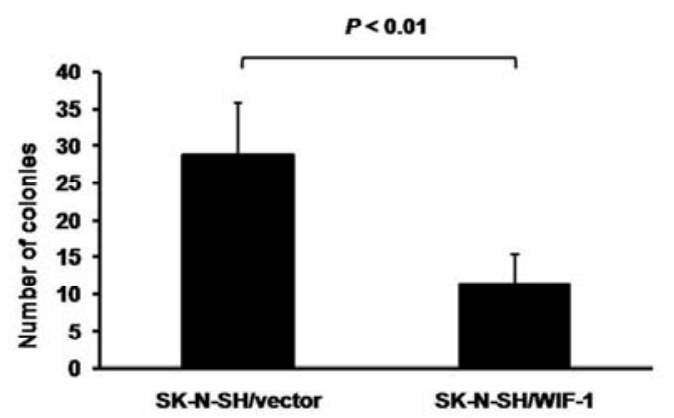

Fig.5. Restoration of WIF-1 inhibits cell growth and proliferation. (A) Cell proliferation curve of SK-N-SH/WIF-1 cells and vector control. Results showed that cell proliferation was inhibited with WIF-1 transfection $(* P<0.01)$. (B) Wnt signaling activity was determined by TOP/FOP flash assays in WIF-1/vector-transfected cells. The colorectal adenocarcinoma cell line Caco2 was used as a positive control. Results showed that Wnt signaling activity was significantly inhibited with WIF-1 transfection $(P<0.05)$. (C) Soft agar colony formation assay was performed in SKN-SH/WIF-1 cells and vector controls. (a: SK-N-SH/vector; b: SK-N-SH/WIF-1). (D) Quantitative analyses of the results of soft agar colony formation assay showed that colony numbers were significantly reduced compared with SK$\mathrm{N}-\mathrm{SH} /$ vector $(P<0.01)$. Mean $\pm \mathrm{SD}$ of three independent experiments.

\subsection{Downregulation of Wnt target gene expression by WIF-1 restoration}

Finally, we examined the expression of Wnt target genes c-myc and cyclin D1. qRT-PCR and Western blotting showed that the expression of c-myc was significantly downregulated in WIF-1-transfected cells compared with vector controls (all $P<0.01$, Figure 3C). ICC analysis showed weaker nuclear staining of c-myc in SK-N-SH/WIF-1 than in negative controls (Supplimental Fig. 1C), and IF analysis showed decreased nuclear inflorescent staining of cmyc in SK-N-SH/WIF-1 compared to negative controls (Fig. 4C).

qRT-PCR and Western blotting also indicated that the expression of cyclin D1 was significantly downregulated in WIF-1-transfected cells compared with vector controls (all $P<0.01$, Fig. 3D). ICC analysis showed weaker nuclear staining of cyclin D1 in SK-NSH/WIF-1 than in negative controls (Supplimental Fig. 1D) and IF analysis showed decreased nuclear inflorescent staining of cyclin D1 in SK-N-SH/WIF-1 than in negative controls (Fig. 4D).

\section{Discussion}

Wnt proteins are thought to be important regulators of cell proliferation and differentiation. Activation of the Wnt/ $\beta$-catenin signaling pathway has been shown to be involved in the pathogenesis of various human tumors [29]. Targeted inhibition of Wnt/ $\beta$-catenin signaling is a 
rational and promising new approach for the therapy of cancers of various origins [30]. As a key antagonistic gene in the Wnt/ $\beta$-catenin signaling pathway, WIF-1 inhibits the upstream of $\mathrm{Wnt} / \beta$-catenin signal transduction by binding to Wnt proteins [13]. Downregulation of WIF-1 expression has been detected in various adult cancers [16-20]. However, the expression and molecular function of WIF-1 remains unknown in pediatric cancer, especially in neuroblastoma.

We demonstrated for the first time that WIF-1 is significantly downregulated in neuroblastoma tissues and cell lines. MSP results showed that there was high level of WIF-1 promoter methylation in neuroblastoma cells. We also investigated the correlation between methylation and WIF-1 downregulation in neuroblastoma. Our data suggests that aberrant hypermethylation of the WIF-1 promoter is an important epigenetic event leading to WIF-1 downregulation in neuroblastoma. Aberrant methylation of promoter regions that silence gene transcription has been recognized as a mechanism for inactivating tumor suppressor genes in some cancers [31, 32]. WIF-1 silencing due to promoter methylation has also been recognized to facilitate tumorigenesis in nasopharyngeal and lung cancer, hepatocellular carcinoma, and colorectal cancer [33-37]. Considering this, we speculate that promoter hypermethylation is the key factor to affect the function of WIF-1 in neuroblastoma.

To further study the potential function of WIF-1 in neuroblastoma, we constructed a WIF1 expression plasmid and transfected it into neuroblastoma cells. We observed that restoration of WIF-1 significantly inhibited neuroblastoma cell growth and colony formation in vitro.

There are previous reports demonstrating that knockdown of the WIF-1 gene significantly increased cell growth $[21,22]$. Others in vivo studies have shown significant inhibition of growth and metastasis formation in WIF-1-transfected xenografts [16, 33, 38]. Taken together, these findings indicate that WIF-1 acts as a tumor suppressor by inhibiting cells growth and proliferation. Considering the close relationship between Wnt/ $\beta$-catenin signaling and WIF-1, we conducted further experiments to verify whether the $\mathrm{Wnt} / \beta$-catenin pathway was involved in the inhibitory effect of WIF-1.

Experiments investigating $\mathrm{Wnt} / \beta$-catenin activity in transfected cells indicated that activation of $\mathrm{Wnt} / \beta$-catenin signaling in $\mathrm{SK}-\mathrm{N}-\mathrm{SH} /$ vector cells was associated with increased luciferase activity of TOP/FOP Flash. We also demonstrated low Wnt/ $\beta$-catenin signaling activity in SK-N-SH/WIF-1 cells, suggesting that restoration of WIF-1 in SK-N-SH cells not only inhibited cells growth and proliferation, but also significantly decreased the activity of $\mathrm{Wnt} / \beta$-catenin signaling at a molecular level. These results suggest a potential role of WIF-1 in inhibiting neuroblastoma cell growth and proliferation.

We further attempted to characterize the expression of $\beta$-catenin, which is a downstream gene in Wnt/ $\beta$-catenin signaling pathway. Previous studies indicate that $\mathrm{Wnt} / \beta$-catenin pathway activation enhances proliferation of several tumor cell lines as a result of increased $\beta$-catenindependent transcription, primarily resulting from $\beta$-catenin protein stabilization [39-41]. In our experiments we demonstrated downregulation of $\beta$-catenin expression in WIF-1 transfected cells at both mRNA and protein levels. These results support the hypothesis that WIF-1 restoration significantly inhibits the activity of $\mathrm{Wnt} / \beta$-catenin pathway and reduces the transmission of $\mathrm{Wnt} / \beta$-catenin signal.

Finally, we examined the expression pattern of the downstream targets of Wnt/ $\beta$-catenin pathway such as c-myc and cyclin D1. Previous studies have shown that c-myc and cyclin D1 can be activated by $\mathrm{Wnt} / \beta$-catenin pathway, and function as proto-oncogenes in many tumors 
[42-44]. We found that both genes were downregulated in response to inactivation of Wnt signaling.

c-myc is a nuclear phosphoprotein that functions as a transcription factor that stimulates cell-cycle progression. It responds directly to mitogenic signals, and plays a critical role in cellcycle progression, especially during transition from G1 to the S phase [18]. Cyclin D1 has a rate-limiting role in G1-S phase transition, and plays a key role in malignant cell proliferation $[45,46]$. Our results indicated that c-myc and cyclin D1 were significantly downregulated by $\mathrm{Wnt} / \beta$-catenin signaling when neuroblastoma cells proliferation was inhibited by WIF-1 restoration. These findings, suggest that WIF- 1 might mediate the function of $\mathrm{Wnt} / \beta$-catenin signaling in neuroblastoma, and that c-myc and cyclin D1 play crucial role in neuroblastoma proliferation.

In summary, we demonstrated that WIF-1 was downregulated by promoter methylation in neuroblastoma. WIF-1 inhibited neuroblastoma cell proliferation and functioned as a tumor suppressor by antagonizing $\mathrm{Wnt} / \beta$-catenin signaling and suppressing the expression of multiple target genes. These findings suggest a potential role for WIF-1 as a therapeutic target in neuroblastoma.

\section{Acknowledgements}

The authors thank Prof. Jie Zhang (Key Laboratory of Transplant Engineering and Immunology, West China Hospital, Sichuan University) for the technical support in the Immunocytofluorescence experiments, and Prof. Rong Wang (Institute of Digestive Surgery, State Key Laboratory of Biotherapy, West China Hospital, Sichuan University) for the technical support in the pathological experiments 


\section{References}

[1] Maris JM. Recent advances in neuroblastoma. N Engl J Med. 2010;362:2202-2211.

[2] London WB, Castleberry RP, Matthay KK, Look AT, Seeger RC, Shimada H, Thorner P, Brodeur G, Maris JM, Reynolds CP, Cohn SL. Evidence for an age cutoff greater than 365 days for neuroblastoma risk group stratification in the Children's Oncology Group. J Clin Oncol. 2005;23:6459-6465.

[3] Taipale J, Beachy PA. The Hedgehog and Wnt signalling pathways in cancer. Nature. 2001;411:349-354.

[4] Morin PJ, Sparks AB, Korinek V, Barker N, Clevers H, Vogelstein B,Kinzler KW. Activation of beta-catenin-Tcf signaling in colon cancer by mutations in beta-catenin or APC. Science. 1997;275:1787-1790.

[5] Weeraratna AT, Jiang Y, Hostetter G, Rosenblatt K, Duray P, Bittner M, Trent JM. Wnt5a signaling directly affects cell motility and invasion of metastatic melanoma. Cancer Cell. 2002;1:279-288.

[6] Uematsu K, He B, You L, Xu Z, McCormick F, Jablons DM. Activation of the Wnt pathway in non small cell lung cancer: evidence of dishevelled overexpression. Oncogene. 2003;22:721872-21.

[7] Robin M. Hallett, Maria K. Kondratyev, Andrew O. Giacomelli, Allison M. L. Nixon, Adele Girgis-Gabardo, Dora Ilieva, John A. Hassell. Small Molecule Antagonists of the Wnt/Beta-Catenin Signaling Pathway Target Breast Tumor-Initiating Cells in a Her2/Neu Mouse Model of Breast Cancer. PLoS One. 2012;7:e33976.

[8] Rubin JS, Barshishat-Kupper M, Feroze-Merzouq F, Xi ZF. Secreted WNT antagonists as tumor suppressors: pro and con. Front Biosci. 2006;11:2093-2105.

[9] Rubin JS, Bottaro DP. Loss of secreted frizzled-related protein-1 expression in renal cell carcinoma reveals a critical tumor suppressor function. Clin Cancer Res. 2007;13:46604663.

[10] Clevers H, Nusse R. Wnt/ $\beta$-catenin signaling and disease. Cell. 2012;149:1192-1205.

[11] Zhi F, Gong G, Xu Y, Zhu Y, Hu D, Yang Y, Hu Y. Activated $\beta$-catenin forces N2A cellderived neurons back to tumor-like neuroblasts and positively correlates with a risk for human neuroblastoma. Int J Biol Sci. 2012;8:289-297.

[12] Hsieh JC, Kodjabachian L, Rebbert ML, Rattner A, Smallwood PM, Samos CH, Nusse R, Dawid IB, Nathans J. A new secreted protein that binds to Wnt proteins and inhibits their activities. Nature. 1999;398:431-436.

[13] Malinauskas T, Aricescu AR, Lu W, Siebold C, Jones EY. Modular mechanism of Wnt signaling inhibition by Wnt inhibitory factor 1. Nat Struct Mol Biol. 2011;18:886-893.

[14] He TC, Sparks AB, Raqo C, Hermeking H, Zawel L, da Costa LT, Morin PJ, Vogelstein $\mathrm{B}$, Kinzler KW. Identification of c-MYC as a target of the APC pathway. Science. 
1998;281:1509-1512.

[15] Tetsu O, McCormick F. Beta-catenin regulates expression of cyclin D1 in colon carcinoma cells. Nature. 1999;398:422-426.

[16] Kawakami K, Hirata H, Yamamura S, Kikuno N, Saini S, Majid S, Tanaka Y, Kawamoto K, Enokida H, Nakagawa M, Dahiya R. Functional Significance of Wnt Inhibitory Factor1 Gene in Kidney Cancer. Cancer Res. 2009;69:8603-8610.

[17] Mazieres J, He B, You L, Xu Z, Lee AY, Mikami I, Reguart N, Rosell R, McCormick F, Jablons DM. Wnt inhibitory factor-1 is silenced by promoter hypermethylation in human lung cancer. Cancer Res. 2004;64:4717-4720.

[18] Rubin EM, Guo Y, Tu K, Xie J, Zi X, Hoanq BH. Wnt Inhibitory Factor 1 decreases tumorigenesis and metastasis in osteosarcoma. Mol Cancer Ther. 2010;9:731-741.

[19] Deng Y, Yu B, Cheng Q, Jin J, You H, Ke R, Tang N, Shen Q, Shu H, Yao G, Zhang Z, Qin W. Epigenetic silencing of WIF-1 in hepatocellular carcinomas. J Cancer Res Clin Oncol. 2010;136:1161-1167.

[20] Wu D, Wong P, Li W, Vogel CF, Matsumura F. Suppression of WIF-1 through promoter hypermethylation causes accelerated proliferation of the aryl hydrocarbon receptor (AHR) overexpressing MCF10AT1 breast cancer cells. Toxicology. 2011; 285:97-103.

[21] Urakami S, Shiina H, Enokida H, Kawakami T, Tokizane T, Ogishima T, Tanaka Y, Li LC, Ribeiro-Filho LA, Terashima M, Kikuno N, Adachi H, Yoneda T, Kishi H, Shigeno K, Konety BR, Igawa M, Dahiya R. Epigenetic inactivation of Wnt inhibitory factor-1 plays an important role in bladder cancer through aberrant canonical Wnt/beta-catenin signaling pathway. Clin Cancer Res. 2006;12:383-391.

[22] Kawamoto K, Hirata H, Kikuno N, Tanaka Y, Nakagawa M, Dahiya R. DNA methylation and histone modifications cause silencing of Wnt antagonist gene in human renal cell carcinoma cell lines. Int J Cancer. 2008;123:535-542.

[23] Schmittgen TD, Livak KJ. Analyzing real-time PCR data by the comparative C(T) method. Nat Protoc. 2008;3:1101-1108.

[24] Yu Vc, Hochhaus G, Chang FH, Richards ML, Bourne HR, Sadee W. Differentiation of human neuroblastoma cells: marked potentiation of prostaglandin E-stimulated accumulation of cyclic AMP by retinoic acid. J Neurochem. 1988;51:1892-1899.

[25] Valentiner U, Valentiner FU, Schumacher U. Expression of CD44 is associated with a metastatic pattern of human neuroblastoma cells in a SCID mousexenograft model. Tumour Biol. 2008;29:152-160.

[26] Reguart N, He B, Xu Z, You L, Lee AY, Mazieres J, Mikami I, Batra S, Rosell R, McCormick F, Jablons DM. Cloning and characterization of the promoter of human Wnt inhibitory factor-1. Biochem Biophys Res Commun. 2004;323:229-234.

[27] Korinek V, Barker N, Morin PJ, van Wichen D, de Weger R, Kinzler KW, Vogelstein B, Clevers H. Constitutive transcriptional activation by a beta-catenin-Tcf complex in APC/- colon carcinoma. Science. 1997;275:1784-1787.

[28] Lüchtenborg M, Weijenberg MP, Roemen GM, de Bruïne AP, van den Brandt PA, Lentjes MH, Brink M, van Engeland M, Goldbohm RA, de Goeij AF. APC mutations in sporadic colorectal carcinomas from The Netherlands Cohort Study. Carcinogenesis. 2004;25:1219-1226.

[29] Karim R, Tse G, Putti T, Scolyer R, Lee S. The significance of the Wnt pathway in the 
pathology of human cancers. Pathology. 2004;36:120-128.

[30] Herbst A, Kolligs FT. Wnt signaling as a therapeutic target for cancer. Methods Mol Biol. 2007;361:63-91.

[31] Katoh H, Yamashita K, Waraya M, Margalit O, Ooki A, Tamaki H, Sakagami H, Kokubo K, Sidransky D, Watanabe M. Epigenetic silencing of HOPX promotes cancer progression in colorectal cancer. Neoplasia. 2012;14:559-571.

[32] Herman JG, Baylin SB. Gene silencing in cancer in association with promoter hypermethylation. N Engl J Med. 2003;349:2042-2054.

[33] Lin YC, You L, Xu Z, He B, Yang CT, Chen JK, Mikami I, Clément G, Shi Y, Kuchenbecker K, Okamoto J, Kashani-Sabet M, Jablons DM. Wnt inhibitory factor-1 gene transfer inhibits melanoma cell growth. Hum Gene Ther. 2007;18:379-386.

[34] Liu YL, Yang HP, Zhou XD, Gong L, Tang CL, Wang HJ. The hypomethylation agent bisdemethoxycurcumin acts on the WIF-1 promoter, inhibits the canonical Wnt pathway and induces apoptosis in human non-small-cell lung cancer. Curr Cancer Drug Targets. 2011;11:1098-1110.

[35] Deng Y, Yu B, Cheng Q, Jin J, You H, Ke R, Tang N, Shen Q, Shu H, Yao G, Zhang Z, Qin W. Epigenetic silencing of WIF-1 in hepatocellular carcinomas. J Cancer Res Clin Oncol. 2010;136:1161-1167.

[36] Lee BB, Lee EJ, Jung EH, Chun HK, Chang DK, Song SY, Park J, Kim DH. Aberrant methylation of APC, MGMT, RASSF2A, and WIF-1 genes in plasma as a biomarker for early detection of colorectal cancer. Clin Cancer Res. 2009; 15:6185-6191.

[37] Batra S, Shi Y, Kuchenbecker KM, He B, Reguart N, Mikami I, You L, Xu Z, Lin YC, Clément G, Jablons DM. Wnt inhibitory factor-1, a Wnt antagonist, is silenced by promoter hypermethylation in malignant pleural mesothelioma. Biochem Biophys Res Commun. 2006;342:1228-1232.

[38] Kim J, You L, Xu Z, Kuchenbecker K, Raz D, He B, Jablons D. Wnt inhibitory factor inhibits lung cancer cell growth. J Thorac Cardiovasc Surg. 2007;133:733-737.

[39] Lu W, Tinsley HN, Keeton A, Qu Z, Piazza GA, Li Y. Suppression of Wnt/beta-catenin signaling inhibits prostate cancer cell proliferation. Eur J Pharmacol. 2009;602:8-14.

[40] Schlange T, Matsuda Y, Lienhard S, Huber A, Hynes NE. Autocrine WNT signaling contributes to breast cancer cell proliferation via the canonical WNT pathway and EGFR transactivation. Breast Cancer Res. 2007;9:R63.

[41] Kaur P, Mani S, Cros MP, Scoazec JY, Chemin I, Hainaut P, Herceg Z. Epigenetic silencing of sFRP1 activates the canonical Wnt pathway and contributes to increased cell growth and proliferation in hepatocellular carcinoma. Tumour Biol. 2012;33:325-336.

[42] Zhang S, Li Y, Wu Y, Shi K, Bing L, Hao J. Wnt/ $\beta$-catenin signaling pathway upregulates c-Myc expression to promote cell proliferation of P19 teratocarcinoma cells. Anat Rec (Hoboken). 2012;295:2104-2113.

[43] Wang C, Tai Y, Lisanti MP, Liao DJ. c-Myc induction of programmed cell death may contribute to carcinogenesis: a perspective inspired by several concepts of chemical carcinogenesis. Cancer Biol Ther. 2011;11:615-626.

[44] Tarapore RS, Siddiqui IA, Saleem M, Adhami VM, Spiegelman VS, Mukhtar H. Specific targeting of $\mathrm{Wnt} / \beta$-catenin signaling in human melanoma cells by a dietary triterpene lupeol. Carcinogenesis. 2010;31:1844-1853. 
[45] Georgopoulou N, Hurel C, Politis PK, Gaitanou M, Matsas R, Thomaidou D. BM88 is a dual function molecule inducing cell cycle exit and neuronal differentiation of neuroblastoma cells via cyclin D1 down-regulation and retinoblastoma protein hypophosphorylation. J Biol Chem. 2006 ;281:33606-33620.

[46] Budhram-Mahadeo VS, Irshad S, Bowen S, Lee SA, Samady L, Tonini GP, Latchman DS. Proliferation-associated Brn-3b transcription factor can activate cyclin D1 expression in neuroblastoma and breast cancer cells. Oncogene. 2008;27:145-154. 
A
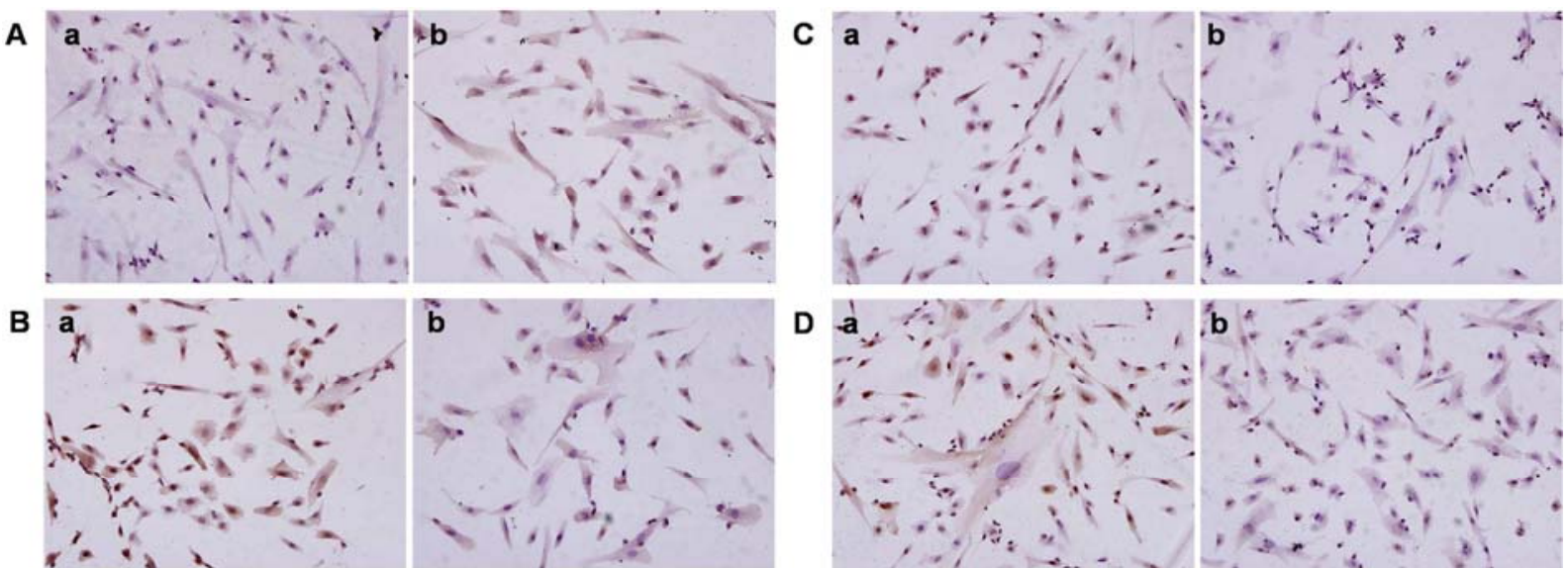

D

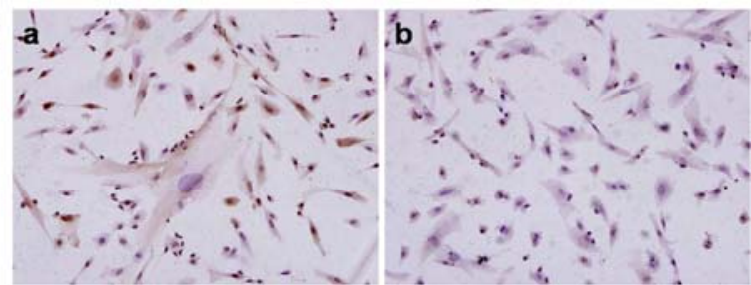

\section{Supplemental Fig. 1}

Immunocytochemistry staining of WIF-1, $\beta$-catenin, c-myc and cyclin D1 in WIF-1/vector transfected SK-N-SH cells ( $\times 200$ magnification; a: SK-N-SH/vector; b: SK-N-SH/WIF-1). (A) WIF-1 expression was upregulated with strong cytoplasmic and membrane staining in SK-N-SH/WIF-1 cells. (B) The expression of $\beta$-catenin was downregulated with weak cytoplasmic and nuclear staining in SK-N-SH/WIF-1 cells. (C) The expression of c-myc was downregulated with weak cytoplasmic and nuclear staining in SK-N-SH/WIF-1 cells. (D) The expression of cyclin D1 was downregulated with weak cytoplasmic and nuclear staining in SK-N-SH/WIF-1 cells.

\section{Supplimental Table 1}

PCR primers sequences used in the MSP and q-RT-PCR.

\begin{tabular}{|c|c|c|c|}
\hline Genes & Primer sequence $\left(5^{\prime}-3^{\prime}\right)$ & $\begin{array}{c}\operatorname{Tm}\left({ }^{\circ} \mathrm{C}\right) \\
\text { PCR cycles }\end{array}$ & $\begin{array}{c}\text { Product size } \\
\text { (bp) }\end{array}$ \\
\hline \multicolumn{4}{|l|}{ WIF-1 MSP Primers } \\
\hline WIF-MF & GGGCGTTTTATTGGGCGTAT & 60,35 & 145 \\
\hline WIF-MR & AAACCAACAATCAACGAAC & & \\
\hline WIF-UF & GGGTGTTTTATTGGGTGTAT & 55,35 & 151 \\
\hline WIF-UR & AAACCAACAATCAACAAAAC & & \\
\hline \multicolumn{4}{|l|}{ qRT-PCR Primers } \\
\hline WIF-F & CCGAAATGGAGGCTTTTGTA & 53,30 & 188 \\
\hline WIF-R & TGGTTGAGCAGTTTGCTTTG & & \\
\hline$\beta$-catenin- $F$ & САТТАСААСТСТССАСААСС & 45,30 & 281 \\
\hline$\beta$-catenin- $R$ & CAGATAGCACCTTCAGCAC & & \\
\hline c-myc-F & GGAGACACCGCCCACCAC & 48,30 & 264 \\
\hline c-myc-R & ACACTGTCCAACTTGACCCT & & \\
\hline cyclin D1-F & CTACACCGACAACTCCATC & 46,30 & 137 \\
\hline cyclin D1-R & CCGCCTCTGGCATTTTGG & & \\
\hline$\beta$-actin- $F$ & AAGGCCAACCGCGAGAA & 48,30 & 166 \\
\hline$\beta$-actin-R & CCTCGTAGATGGGCACA & & \\
\hline
\end{tabular}

Note. M: methylation-specific primer; U: unmethylation-specific primer; F: forward primer; R: reverse primer. 